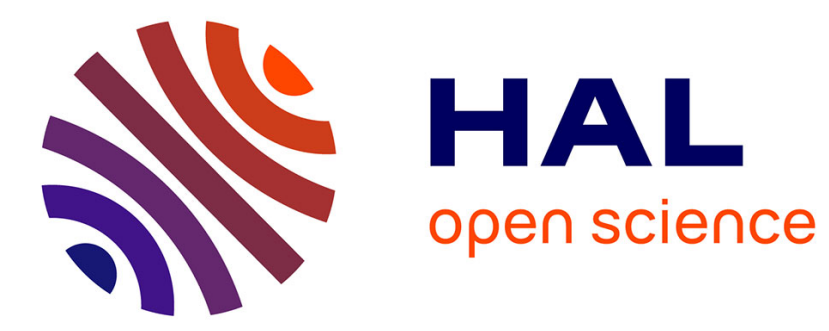

\title{
Les migrants à Hanoi : Construction politique d'un groupe social dominé
}

\author{
Gwenn Pulliat
}

\section{To cite this version:}

Gwenn Pulliat. Les migrants à Hanoi : Construction politique d'un groupe social dominé. Espaces et sociétés (Paris, France), 2013, 154, pp.87-102. 10.3917/esp.154.0087 . hal-01453474

\section{HAL Id: hal-01453474 \\ https://hal.science/hal-01453474}

Submitted on 6 Feb 2017

HAL is a multi-disciplinary open access archive for the deposit and dissemination of scientific research documents, whether they are published or not. The documents may come from teaching and research institutions in France or abroad, or from public or private research centers.
L'archive ouverte pluridisciplinaire HAL, est destinée au dépôt et à la diffusion de documents scientifiques de niveau recherche, publiés ou non, émanant des établissements d'enseignement et de recherche français ou étrangers, des laboratoires publics ou privés. 


\title{
LES MIGRANTS À HANOÏ : CONSTRUCTION POLITIQUE D'UN GROUPE SOCIAL DOMINÉ
}

\author{
Gwenn Pulliat
}

ERES | Espaces et sociétés

\author{
2013/3 - n 154 \\ pages 87 à 102
}

\section{ISSN 0014-0481}

Article disponible en ligne à l'adresse:

http://www.cairn.info/revue-espaces-et-societes-2013-3-page-87.htm

Pour citer cet article :

Pulliat Gwenn, «Les migrants à Hanoï : Construction politique d'un groupe social dominé », Espaces et sociétés, 2013/3 n 154, p. 87-102. DOI : 10.3917/esp.154.0087

Distribution électronique Cairn.info pour ERES.

(c) ERES. Tous droits réservés pour tous pays.

La reproduction ou représentation de cet article, notamment par photocopie, n'est autorisée que dans les limites des conditions générales d'utilisation du site ou, le cas échéant, des conditions générales de la licence souscrite par votre établissement. Toute autre reproduction ou représentation, en tout ou partie, sous quelque forme et de quelque manière que ce soit, est interdite sauf accord préalable et écrit de l'éditeur, en dehors des cas prévus par la législation en vigueur en France. II est précisé que son stockage dans une base de données est également interdit. 


\title{
Les migrants à Hanoï : Construction politique d'un groupe social dominé
}

\section{Gwenn Pulliat}

\begin{abstract}
Avec la mise en place du Đổi Mó́i (Renouveau) à partir de 1986, le Vietnam a choisi un développement économique fondé sur l'ouverture au commerce international et l'industrialisation du pays. Les investissements sont prioritairement dirigés vers les grandes villes, ce qui se traduit par un mouvement d'urbanisation rapide : de 1989 à 2009, la population urbaine a plus que doublé, alors que la population totale a augmenté d'un tiers (Quertamp, 2010 ; GSO $^{1}$, données 2011). Le taux de population urbaine est passé, selon les statistiques officielles, de $19 \%$ en 1989 à $30 \%$ aujourd'hui : c'est une rupture importante par rapport à la volonté politique qui prévalait jusqu'alors de limiter les migrations vers la ville et de favoriser la redistribution de la population vers les zones les moins densément peuplées du territoire.
\end{abstract}

Gwenn Pulliat, doctorante en géographie, Laboratoire Mosaïques, UMR 7218 Lavue, CNRS et Université Paris Ouest gwenn.pulliat@gmail.com

1. Office général de la statistique, site : http://www.gso.gov.vn. 
Le processus de transition urbaine ainsi engagé (Quertamp, op. cit.; Castiglioni et al., 2006) produit une structuration du territoire qui s'articule autour des deux métropoles du pays : Hanoï et Hô Chi Minh Ville. Hô Chi Minh Ville, au Sud, est la plus grande cité du pays et le principal pôle d'attractivité économique. Hanoï se trouve à la tête de l'armature urbaine de la moitié nord: elle polarise les activités économiques, assure une domination politique et se trouve au centre des dynamiques migratoires de la région Nord du pays. En devenant le point de convergence des flux de biens, de capitaux, de personnes, elle est entrée dans un processus de métropolisation (Lacoste, 1993 ; Bassand et al., 2000) : l'intégration économique internationale a donné l'impulsion. Ce phénomène procède à la fois d'une dynamique spontanée liée aux choix des investisseurs étrangers (qui représentent $30 \%$ des investissements totaux en 2008, OCDE, 2011) et d'une stratégie politique visant à faire de Hanoï une capitale d'ampleur internationale (Labbé et Musil, 2011). La refonte des limites administratives en $2008^{2}$ et le plan de développement à l'horizon 2030, voté en 2011, témoignent de cette volonté de l'État de faire de Hanoï une vitrine pour le pays et une métropole d'échelle régionale ${ }^{3}$.

Le corollaire de ce processus de métropolisation est l'essor des migrations vers la capitale. Les migrations de l'hinterland vers Hanoï ne sont pas nouvelles : la ville a longtemps fait office de marché où les villageois des campagnes du delta du fleuve Rouge échangeaient leurs produits. Mais l'instauration d'un fort contrôle des déplacements à partir de 1955 a mis un terme à l'intense va-et-vient, qui prévalait jusqu'alors (Papin, 2001) entre Hanoï et son hinterland. Ce contrôle se fondait sur l'existence d'un livret de famille (hộ khẩu) associé à un lieu de résidence et sur l'obligation de déclarer tout déplacement au départ et à l'arrivée. Ces dispositifs existent toujours, mais la contrainte a considérablement diminué avec la suppression, en 1989, des coupons alimentaires qui étaient associés au hộ khẩu, puis l'assouplissement des conditions d'obtention d'un permis de résidence permanent par la loi sur la résidence de 2006. Ainsi les migrations - qu'elles soient définitives ou saisonnières - se sont accélérées, facilitées par l'amélioration des transports.

2. Les motifs sont multiples : stratégie d'image, mais également spéculation foncière et prise de contrôle par les autorités de Hanoï de l'ensemble de l'aire d'expansion de la capitale - limitée jusqu'alors à la province de Ha Tay (Labbé \& Musil, op. cit.).

3. Capitale administrative, Hanoï attire moins les investisseurs internationaux que Ho Chi Minh Ville ; l'État vietnamien, concentré à Hanoï, le pouvoir entend rééquilibrer l'attractivité en faveur du Nord. 
L'ampleur des migrations est difficile à évaluer. Si l'on peut connaître les changements de hô khẩu et, dans une certaine mesure, les migrants avec un permis de résidence temporaire installés durablement en ville, aucune statistique n'existe sur les migrations de court terme, les migrations saisonnières, les migrations « circulaires » (caractérisées par un retour régulier au lieu de départ, qui reste le lieu de résidence, Brunet, 1992). Patrick Gubry et al. (2011) soulignent le caractère fantaisiste de la plupart des données sur la population urbaine de Hanoï ; ils insistent sur l'absence de données fiables concernant ce que l'on désigne sous le terme générique de « migrants ». Cette catégorie administrative désigne les porteurs d'un enregistrement temporaire (se trouvant donc dans une autre province que celle où est établi leur $h \hat{o}$ khẩu); elle regroupe en réalité une population très variée, avec des personnes de passage en ville pour une courte durée, des personnes qui font des allers-retours réguliers entre Hanoï et leur lieu d'origine et des personnes qui résident de fait à Hanoï mais n'ont pas la possibilité d'y faire établir leur hộ khẩu, pour des raisons évoquées plus loin. Elle regroupe des visiteurs, des « migrants » installés récemment à Hanoï et ayant vocation à y rester, et une « population flottante » aux pratiques migratoires diverses. Aucune statistique ne permet d'évaluer aujourd'hui l'ampleur de ces phénomènes migratoires.

Dans les discours quotidiens, on associe le vocable de « migrants » à cette « population flottante », d'origine rurale et travaillant en ville, mais sans possibilité de devenir résidente. Ainsi nommée, elle est perçue comme un groupe social homogène, associé à un statut social inférieur. Le terme est approprié par les « migrants » eux-mêmes, qui se désignent ainsi par opposition aux « Hanoïens ». Comment cette catégorisation administrative - qui distingue « résidents » et « migrants » sur la base de l'enregistrement résidentiel construit-elle une minorité urbaine (en tant que groupe partageant un certain nombre de caractères propres, identifié comme groupe particulier par les autres citadins et se percevant elle-même comme telle) et entérine-t-elle des rapports sociaux de domination? 


\section{MÉTHOdologie}

Cet article se fonde sur un ensemble d'entretiens réalisés en 2011 dans trois quartiers de Hanoï. Le premier, Phúc Tân, est géographiquement central, mais se situe entre la digue et le fleuve Rouge : c'est une zone inondable. Le second, Yên Sở, se trouve à la limite sud de Hanoï : il était jusqu'en 2004 classé comme quartier rural, mais fait désormais l'objet d'une urbanisation rapide, avec plusieurs projets d'infrastructures (autoroute, parc urbain, zone industrielle...). Le dernier enfin, An Khánh, situé à $8 \mathrm{~km}$ à l'ouest de Hanoï, est un village périurbain dont le territoire est traversé depuis 2010 par une importante autoroute; toutes les terres agricoles ont été expropriées afin de réaliser une grande zone d'habitation et une zone industrielle. Ces projets sont en cours de réalisation.

Les entretiens de la première série sont semi-directifs, avec l'objectif de restituer les trajectoires professionnelles, spatiales et familiales des enquêtés, mais aussi leur perception de l'espace dans lequel ils vivent. L'objectif est de cibler la population défavorisée de Hanoï (pas exclusivement les «migrants ») ; les principaux critères de sélection sont la précarité du logement et des formes d'emploi. Nos entretiens présentent une large diversité de situations permettant de voir les différentes pratiques quotidiennes. Nous disposons de 32 entretiens de "migrants", parmi lesquels 20 « flottants » vivant à la fois à la ville et à la campagne (présents en moyenne depuis 12 ans à Hanoï, ils ne sont pas juste de passage) et 12 personnes ayant un hộ khẩu dans une autre province mais résidant de fait à Hanoï. Nous disposons également d'une dizaine d'entretiens de résidents louant des chambres à des « migrants".

La seconde série d'entretiens s'adresse aux autorités locales, à plusieurs niveaux : présidents ou vice-présidents des Comités populaires des districts et des quartiers ${ }^{4}$, chefs d'îlots, mais aussi responsables des sections locales des grandes associations nationales (para-étatiques), notamment l'Union des femmes et la Croix-Rouge. 20 entretiens ont été réalisés, avec l'objectif de mettre en évidence la façon dont ces autorités locales perçoivent les enjeux, l'évolution et les problèmes quotidiens des quartiers étudiés - dont la place des « migrants ». Le but est de confronter ces perceptions de la vie locale avec l'espace vécu par ses habitants.

4. La province de Hanoï est divisée en 29 districts, eux-mêmes subdivisés en quartiers (ou communes dans le cas des districts ruraux). Les quartiers constituent l'unité administrative de base ; mais ils sont ensuite découpés en " îlots » de population, gérés par des chefs d'îlot, qui assurent l'encadrement de la population pour toutes les tâches quotidiennes (gestion des conflits de voisinage, information et aide dans les tâches administratives etc.). 


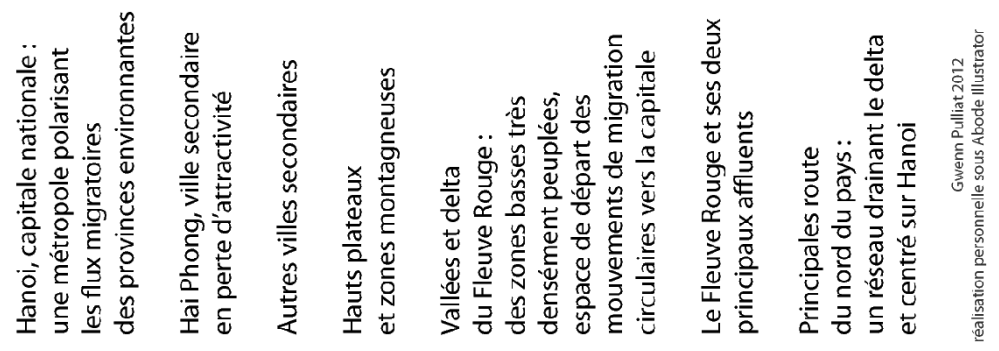

:
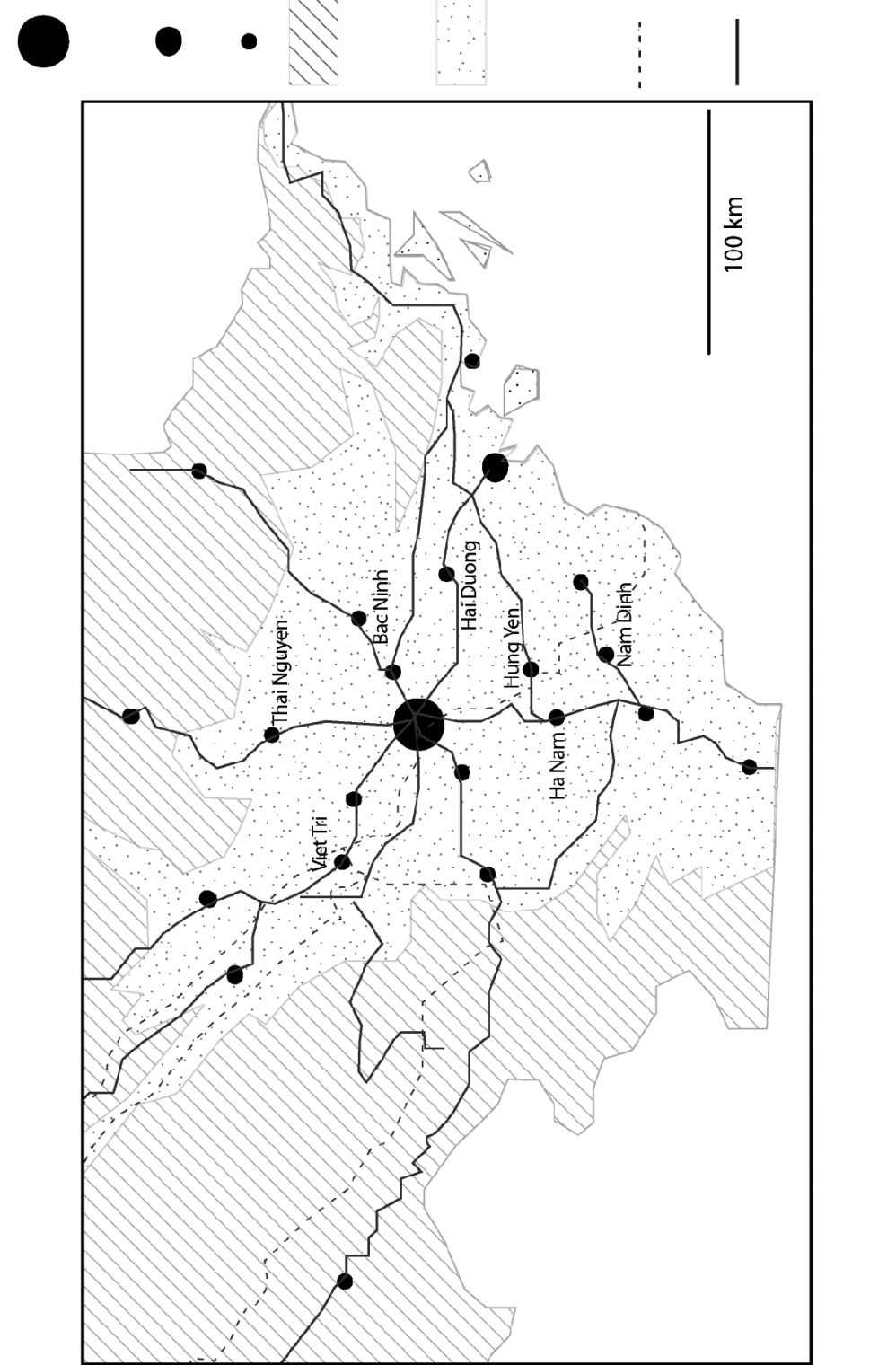

ำ

을

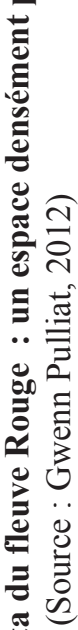

है

Uั 


\section{MÉTROPOLISATION, ATTRACTIVITÉ URBAINE ET MIGRATIONS}

\section{La métropolisation : polarisation économique et mobilité spatiale croissante}

Le terme de «métropolisation» est défini par Roger Brunet (op. cit.) comme un "mouvement accusé de concentration de la population dans les métropoles » : c'est le phénomène humain, donc migratoire, qui le caractérise. Le fait métropolitain se traduit par une concentration spatiale des activités économiques, des centres de pouvoir, des services supérieurs (Lacoste, op. cit.), qui induit une attraction forte sur un territoire à large échelle. Hanoï exerce son influence sur la région Nord du pays, d'où vient la quasi-totalité des migrants vers la capitale (VeT, 2005 ${ }^{5}$ ). Le différentiel de revenus attendus entre le milieu rural et Hanoï explique largement la mobilité croissante de la maind'œuvre rurale, qui vient chercher en ville un revenu principal ou complémentaire. Le différentiel est d'autant plus attractif que la mobilité est facilitée par des coûts de transport faibles grâce au réseau de bus et par la présence en ville de logements peu onéreux (même s'ils sont précaires).

\section{Les migrants : une population hétérogène}

L'attractivité de la métropole se traduit de façon variable dans les pratiques migratoires. S'il y a un mouvement de concentration de la population dont témoignent les migrations définitives, on observe également d'importants mouvements de plus courte durée, avec maintien d'une vie à la campagne. La présence en ville peut constamment s'adapter aux besoins et aux opportunités de la famille des migrants. La migration permet une diversification des ressources, mobilisées dans des espaces différenciés. Pour cette raison, le processus de métropolisation autour de Hanoï ne se traduit pas par un exode rural massif, mais souvent par une circulation accrue des personnes, des biens, des ressources, tirant parti de la complémentarité des deux espaces, sans rupture majeure. Les bénéfices réalisés à Hanoï s'investissent souvent à la campagne : pour ces personnes il s'agit de "partir pour rester» (Racine, 1994 ; Cortès, 2000), de migrer pour assurer un maintien ou une amélioration des conditions de vie de la famille à la campagne.

« Je fixe un budget de 15000 vND $^{6}$ par jour pour me nourrir et je ne veux pas dépasser, parce qu'il faut économiser le plus d'argent possible, sinon il ne me reste plus assez à envoyer. C'est nécessaire pour payer l'école des deux petits-enfants,

5. Hô Chi Minh Ville accueille des migrants venus de l'ensemble du pays, y compris du Nord. 6. Soit environ $0,55 €$, représentant dans son cas de l'ordre de $10 \%$ de ses revenus quotidiens. 
parce que mon fils ne gagne pas assez à la campagne. Et puis un autre petit-fils a eu un accident récemment, j'ai donné tout mon argent pour aider à payer l'hôpital » ( $\mathrm{M}^{\mathrm{me}}$ Chinh, 62 ans, originaire de la province de Hung Yen et venant à Hanoï depuis 31 ans).

La comparaison des pratiques migratoires entre nos enquêtés les plus pauvres et les plus aisés, rejoignant Gubry et al. (2008), suggère qu'un net accroissement des revenus en ville, ainsi que la sécurisation de l'emploi, conduisent à une rupture plus franche, produisant un glissement de la mobilité vers la migration définitive.

La variété des formes migratoires accompagne la variété des profils des migrants. S'il y a généralement un motif économique à l'origine de la venue à Hanoï, tous les migrants ne sont pas pauvres, ni au départ, ni à l'arrivée ${ }^{7}$. L'enquête sur la pauvreté urbaine menée par le PNUD (2010), montre qu'en moyenne les migrants ont des revenus un peu inférieurs pour un temps de travail plus long, une formation moindre et des emplois moins qualifiés, des formes d'emploi très souvent précaires (sans contrat de travail, ou avec des contrats de courte durée) : les statuts d'emploi inférieurs accueillent une proportion plus importante de migrants que les emplois valorisés. Mais le groupe des migrants ne se limite pas à cette population précaire : il y a aussi des étudiants venus d'autres régions, trouvant ensuite des emplois stables et bien rémunérés, des personnes qualifiées venues se faire embaucher dans de grandes entreprises, des fonctionnaires en mutation, etc. Gubry et al. (2008) ont mis en évidence les conditions de vie plutôt favorables de ces migrants qui s'installent souvent de façon définitive dans la métropole. Un revenu plus élevé permet, à terme, d'obtenir un enregistrement résidentiel permanent à Hanoï : ces migrants sont des résidents en devenir, alors que les migrants précaires, évoqués précédemment, n'ont guère la possibilité de changer de catégorie administrative. Cela explique que les représentations sociales associent le terme de « migrants » à ces derniers, quelle que soit la réalité de leur présence en ville.

La catégorie de «migrants » désigne majoritairement une population d'origine rurale, mais sans distinction selon le rapport à la ville. Il y a d'une part des personnes qui conservent un ancrage à la campagne et qui circulent entre les deux espaces, au gré des contraintes et des opportunités qui se présentent, adoptant une vie multi-locale. Le terme de « population flottante », utilisé en Chine pour désigner les personnes ayant un livret de résidence (hukou) rural et qui s'installent en ville (Li Z., 2001), paraît particulièrement adapté au cas

7. Même quand ils sont sous le seuil de pauvreté, les migrants estiment avoir une espérance de gains à Hanoï, supérieure à ce qu'ils percevraient à la campagne. L'étude du groupe Villes en Transition (VeT, 2005) conclut que $95 \%$ des migrants estiment que leur vie s'est améliorée après la migration. 
des métropoles vietnamiennes (Luong et al., 2011 ; Gubry et al., 2011). Il y a d'autre part les ruraux installés définitivement en ville, mais sans pouvoir obtenir un enregistrement résidentiel à Hanoï. Le terme de « migrants », tel qu'utilisé par les autorités comme par les résidents de Hanoï, amalgame ces deux situations : il s'agit des personnes ayant un enregistrement résidentiel dans une autre province, sans avoir la possibilité de devenir « résidentes » à Hanoï à court ou moyen terme.

\section{L'ENREGISTREMENT RÉSIDENTIEL : CATÉGORIES ADMINISTRATIVES ET DROITS SOCIAUX DIFFÉRENCIÉS}

\section{À quoi sert l'enregistrement résidentiel?}

Dès l'indépendance en 1954, l'État socialiste a cherché à contrôler le développement de la capitale (Papin, op. cit.) et à organiser le peuplement du pays. L'armature administrative permettant de contrôler la mobilité des personnes ( $h \hat{o}$ khẩu et enregistrement des déplacements auprès de la police) s'est progressivement assouplie : la libéralisation économique nécessite une circulation plus aisée de la main-d'œuvre. Il s'agit d'une réponse à la transition économique engagée avec le Đổi Mới. Si les autorités entendent conserver un certain contrôle sur la population en maintenant cette obligation d'enregistrement, le développement des permis de résidence temporaire et la suppression des contraintes fortes qui pesaient dessus (comme la quasi-impossibilité de scolariser des enfants ailleurs que dans leur commune d'enregistrement, ou d'obtenir un contrat d'électricité ou d'eau courante sans hộ khẩu local) autorisent la circulation des personnes à travers le pays.

\section{Enregistrement résidentiel et droits différenciés}

Bien qu'il n'entrave plus les déplacements, l'enregistrement résidentiel reste associé à des droits sociaux différenciés. Il distingue d'abord citadins et ruraux ; en milieu rural, il ouvre des droits fonciers qui n'existent pas en ville : le droit de bénéficier des terrains décollectivisés par la loi foncière de 1993. Ces terres jouent un rôle important dans l'économie familiale, à plusieurs titres :

- quand elles sont cultivées par leur propriétaire ou sa famille, elles assurent un approvisionnement alimentaire qui représente un filet de sécurité. Elles assurent aussi parfois un revenu complémentaire, surtout pour les " inactifs » du foyer (personnes âgées) ;

- elles représentent un potentiel économique pour la famille : elles sont une caution qui facilite l'obtention d'un prêt auprès d'une institution financière ; - c'est un capital cessible aux enfants, ce qui a souvent été évoqué au cours de nos enquêtes, par des ménages qui avaient été expropriés de leurs terres : ils déploraient ne plus avoir ce capital productif dont les enfants auraient dû hériter ; 
- enfin, en cas d'expropriation, elles deviennent une ressource financière importante ${ }^{8}$. Même en cas de migration, les propriétaires cherchent à affirmer leur présence (les terres non cultivées revenant théoriquement aux autorités publiques) en les prêtant à un membre de la famille ou un voisin, afin de bénéficier des potentielles retombées économiques ultérieures.

Une image de sécurité économique est associée à ces terres agricoles. Par conséquent, le changement de lieu d'enregistrement de la campagne vers la ville a un coût d'opportunité non négligeable, puisqu'il implique la perte de ces droits fonciers : c'est un facteur important dans la décision de changer ou non de lieu de résidence officielle.

L'enregistrement résidentiel distingue ensuite migrants et résidents. Le $h \hat{o}$ $k h a ̂ ̉ u$ est associé aux droits sociaux, auxquels les migrants n'ont pas accès en ville. Toutes les démarches administratives et les aides en dépendent: les crédits à taux préférentiel proposés par les associations para-étatiques, le statut de foyer pauvre qui ouvre droit à une assurance santé gratuite et à une réduction des frais de scolarité, etc. Sans hô khẩu local, les familles peuvent scolariser leurs enfants mais en payant des frais supplémentaires importants. En conséquence, si l'enregistrement résidentiel ne représente plus une barrière réelle à la migration, il continue de dessiner deux catégories de citoyens hiérarchisées et il fonde une légitimité différenciée à être en ville.

\section{Une classification administrative source d'inégalités sociales}

Cette hiérarchisation se mue en inégalités sociales quand on examine les critères d'obtention d'un hộ khẩu local. Si la conservation d'un hộ khẩu à la campagne est un choix du migrant, il s'agit souvent d'une contrainte (surtout pour les personnes qui n'ont plus de lien avec leur lieu d'origine). Même si la loi de 2006 a simplifié les démarches, il y a toujours trois conditions à remplir pour obtenir un enregistrement permanent urbain : habiter dans le quartier depuis au moins un an, avoir un emploi régulier et justifier d'un logement stable et légal. Pour les Comités populaires de quartier, qui sont en charge de l'examen des dossiers, la stabilité du logement signifie la propriété, celle de l'emploi est actée par la présence d'un contrat de travail de longue durée. Dès lors, ceux qui ont la possibilité de faire changer leur enregistrement sont les personnes qui ont les moyens financiers d'acquérir un logement et bénéficient de conditions d'emploi sécurisées. Ces conditions excluent d'office toutes les personnes qui travaillent dans le secteur informel - même si, en pratique, le critère de la propriété du logement est le plus important. C'est précisément le

8. À An Khánh, de l'aveu des autorités locales, les indemnités ont été assez basses, l'expropriation d'un sao $\left(360 \mathrm{~m}^{2}\right)$ a été indemnisée à hauteur de 46 millions de dongs (environ $\left.1800 €\right)$, soit grossièrement 3 à 4 ans de salaire moyen par personne. 
secteur informel qui accueille une large partie des migrants, notamment parce que les types d'activités qu'on y trouve (vente, manutention, services à la personne etc.) permettent généralement l'embauche d'une main-d'œuvre sans qualification particulière.

Ces pratiques administratives fondent une inégalité dans l'accès aux services publics et approfondissent les inégalités entre ceux qui disposent d'un enregistrement permanent et ceux qui n'en ont pas, mais aussi entre des migrants qui acquièrent un statut socio-économique favorisé et peuvent devenir « résidents », et ceux qui demeurent précaires et sont contraints de rester dans une situation d'enregistrement temporaire quel que soit leur lieu d'habitation réel. Elles renforcent la différenciation sociale entre les « résidents » actuels et en devenir, considérés comme de véritables citadins, et ces « migrants » qui n'obtiendront jamais le statut de citadins et qui demeurent dans une situation économique et sociale précaire.

\section{Le terme de "migrant " : un étiquetage source d'infériorisation}

Le terme de « migrant » fonctionne comme un étiquetage de cette population d'origine rurale qui est plus tolérée que réellement acceptée en ville. Son usage traduit un rapport de domination entre des résidents légitimes et des « migrants » qui le seraient moins - rapport qui s'exprime au quotidien par la discrimination sociale et les insultes régulières dont sont victimes les " migrants ». Comme en Chine, ils sont perçus comme source de maux sociaux : Li T. (1996) rapporte que les autorités publiques estiment que $80 \%$ des prostituées en ville sont des femmes d'origine rurale; nos enquêtes montrent l'association fréquente faite, par les résidents comme par les autorités publiques, entre « migrants » et trafic de drogue, vols, jeux d'argent. C'est bien un discours d'infériorisation que l'on entend :

«Les migrants, c'est l'un des inconvénients du quartier, parce que ce sont surtout eux qui font des jeux d'argent, des paris et qui sont drogués » (président du Comité populaire de Phúc Tânà).

« Ici, les autorités locales n'encouragent pas les migrants à venir, mais on ne peut pas les en empêcher non plus. Donc on les contrôle strictement, pour être sûr qu'ils ne fassent pas de trafic de drogue etc. On appelle la police en cas de doute, pour vérifier. C'est indispensable pour assurer la sécurité du quartier » (un chef d'îlot de Phúc Tân).

Dans les quartiers périphériques et villages périurbains connaissant une forte croissance démographique, l'arrivée de personnes extérieures au quartier est également perçue comme une source d'insécurité croissante : à Yên Sở par exemple, un chef d'îlot associe à cette population l'accroissement des vols de motos. La dépréciation se poursuit en leur attribuant des comportements jugés négatifs, dégradants pour le quartier, voire dangereux pour les habitants : 
«Les gens qui viennent de la campagne n'ont pas l'esprit des urbains : par exemple, ils jettent les déchets dans la rue, les vendeurs d'aliments ne respectent pas les règles d'hygiène » (chef d'îlot, déjà cité).

«La plupart des migrants sont de passage, ou sont là de façon irrégulière. Pourtant ce serait bien qu'ils suivent eux aussi les formations sur les règles d'hygiène, la gestion des déchets, le respect de l'environnement... Ce sont des recommandations qui ne sont pas suivies par les migrants, alors qu'ils travaillent souvent dans la vente ambulante et ce genre de choses » (la responsable de l'association de la Croix Rouge de Phúc Tân).

\section{« MigRANTS » ET RÉSIDENTS : DES RAPPORTS SOCIAUX DE DOMINATION}

\section{" Migrants " et résidents : des relations économiques intenses et hiérarchisées}

C'est généralement pour chercher une activité économique que les ruraux viennent s'installer en ville (Agergaard et $\mathrm{Vu}, 2010)$. Ils occupent majoritairement des emplois sans aucune sécurité. Quelques types d'emploi semblent prédominer, avec des degrés de formalisation variables. Dans le secteur informel, il y a toutes les activités de vente et de service : vente ambulante, à pied ou à vélo, constituant l'image d'Épinal des « migrants », récupération des déchets valorisables, manutention, moto-taxi, etc. Ces travaux s'effectuent à la tâche et sont rémunérés de la même façon. C'est aussi le cas pour les personnes qui se font embaucher sur des chantiers de construction sans être affiliées à un groupe de travail régulier. À un degré supérieur de formalisation des activités, comme l'emploi dans des restaurants, des magasins et petits ateliers, l'embauche se fait sur un terme plus long, mais sans contrat de travail, avec des salaires bas, sans sécurité. Dans les nouvelles zones industrielles, la formalisation plus grande de l'emploi s'accompagne d'un degré supérieur de sécurité : ils bénéficient d'un contrat de travail, mais le plus souvent de courte durée (souvent 3 mois) renouvelé à plusieurs reprises. L'obtention d'un contrat à durée indéterminée s'accompagne d'une forme d'ascension sociale, d'une installation durable en ville et de la sortie du groupe des «migrants ». Cette typologie des formes d'emploi révèle aussi que les « migrants » entretiennent un partenariat économique avec des résidents : il n'y a pas d'économie «migrante» parallèle. Toutefois, la hiérarchie sociale est corrélée à la hiérarchie économique.

\section{Migrations et réseaux de solidarité villageois : standardisation des conditions de vie en ville}

Les activités exercées par la population flottante présentent certaines particularités qui s'expliquent par les modes d'entrée dans la ville. La migration est rarement un acte isolé : le plus souvent, le migrant s'appuie sur un réseau de relations et suit un proche déjà installé à Hanoï. Des réseaux 
d'entraide familiaux et villageois se créent ainsi : le nouveau venu est aidé pour trouver un logement, puis son aide l'introduit dans l'activité économique qu'il exerce. Souvent les personnes d'un même village, venues successivement, partagent leur lieu de vie et exercent les mêmes activités. L'origine est un critère essentiel dans la construction des réseaux :

« Je suis venue avec une amie du village qui venait depuis 2 ou 3 mois. Au début, on partageait une chambre toutes les deux, puis on a rencontré d'autres personnes du village qu'on ne connaissait pas et qui nous ont proposé de partager leur colocation. On habite toutes ensemble depuis. C'est bien, je préfère vivre avec des gens du village plutôt que d'ailleurs, on se fait confiance et il n'y a pas de problème, personne ne vole les affaires des autres » ( $\mathrm{M}^{\mathrm{me}}$ Nôi, 50 ans, vendeuse ambulante de produits d'hygiène, originaire de Nam Đinh, vient à Hanoï depuis 16 ans).

Les migrants créent des réseaux d'emploi où l'entraide joue à plein, avec un partage d'information (sur les lieux de vente favorables ou les contrôles de police par exemple) voire une mutualisation des achats (denrées à vendre achetées en gros, diable à partager pour les manutentionnaires, etc.). Ces réseaux ne sont pas institutionnalisés, ils se fondent sur l'origine géographique commune et favorisent l'homogénéisation des activités des migrants.

Les conditions de vie de la population flottante présentent certaines spécificités. Les migrants n'étant pas propriétaires de leur logement (puisque cela ouvrirait droit à l'obtention d'un enregistrement permanent local), ils sont le plus souvent locataires, ou parfois hébergés par de la famille, ou encore ils vivent sur le lieu de travail (dans les boutiques ou les ateliers par exemple). C'est une différence majeure avec les résidents, dont plus de $80 \%$ sont propriétaires (ou de la famille du propriétaire) du logement qu'ils habitent (données GSO, 2010 ; PNUD, 2010). Deux formes d'habitat prédominent chez les migrants. La première est la plus économique : la location d'une chambre chez des résidents, soit individuellement, soit partagée entre plusieurs personnes. Parmi nos enquêtés, plus de la moitié des migrants se logent de la sorte, et parmi eux la moitié n'a pas accès à la cuisine. Les surfaces sont minuscules : souvent moins de $7 \mathrm{~m}^{2}$ par personne, dans nos observations. C'est une forme de logement précaire, mais peu onéreuse et flexible, souvent payée à la nuit. La seconde forme se développe rapidement dans les zones périphériques de la ville : des chambres construites pour la location, qui présentent une morphologie particulière (souvent un bâtiment de béton brut avec trois ou quatre pièces en enfilade, de plain-pied, avec des sanitaires partagés) imprimant ainsi leur marque dans le paysage urbain. C'est une forme d'habitat particulière immédiatement identifiable comme logements de migrants, car rares sont les résidents qui y demeurent ${ }^{9}$. On assiste ainsi à une standardisation des formes d'habitat pour les migrants.

9. Dans les deux cas rencontrés de résidents dans ce type de logement, il s'agissait d'un descendant du propriétaire qui vivait là à titre gratuit et en attendant d'avoir épargné pour acquérir une maison individuelle. 
Cette homogénéisation des emplois et des formes d'habitat favorise le processus d'étiquetage et d'identification par les résidents en tant que « migrant ». En associant ces conditions de vie au groupe des migrants et en favorisant l'entre-soi dans la vie quotidienne, elle conduit à l'intériorisation de l'identité de « migrants ». Ces caractères amènent les agents à se représenter comme appartenant à cette minorité, quelle que soit leur situation migratoire réelle. Ils opposent le « nous » des personnes précaires d'origine rurale aux «Hanoïens » qui seraient les « vrais » citadins : c'est ce dont témoignent plusieurs de nos enquêtées :

«Au début où j'étais à Hanoï, les gens entendaient mon accent provincial, ils voyaient que je m'habillais comme une campagnarde, alors ils se moquaient souvent de moi et on me manquait de respect. La plupart des gens ne respectent pas les migrants. Les Hanoïens pensent que ce sont des pauvres, des gens peu cultivés et qu'ils ne méritent pas d'être respectés » ( $\mathrm{M}^{\mathrm{me}}$ Thuy, 27 ans, originaire de Hai Duong, à Hanoï depuis 13 ans, employée dans un atelier de bijouterie).

« Je ne fréquente personne d'autre que mes colocataires, parce que les Hanoïens se moquent souvent de nous, les migrants, certains sont méprisants, nous parlent mal, parfois nous insultent. Bien sûr que je suis une campagnarde, mais ce n'est pas une raison, je n'aime pas du tout les gens qui me parlent mal comme ça » ( $\mathrm{M}^{\mathrm{me}}$ Nôi, 50 ans).

\section{Une mixité sociale paradoxale}

Les migrants logent principalement chez des résidents, ou à proximité immédiate du logement du propriétaire : il en découle une mixité résidentielle entre les deux groupes. On trouve des chambres à louer dans toute la ville et il n'y a pas de quartiers exclusivement réservés à l'accueil de migrants ruraux comme en Chine (Thireau et Linshan, 2002). Il n'y a pas de relégation des zones d'habitat pour migrants à la périphérie de la ville, même dans les quartiers où les logements pour migrants sont nombreux, on observe une certaine diversité des statuts résidentiels et des situations socioéconomiques des habitants (données statistiques à l'échelle des quartiers des districts étudiés). Si les formes typiques d'habitat migrant sont immédiatement identifiables, elles demeurent inscrites dans le tissu urbain et se mêlent aux habitations traditionnelles. Si le processus de métropolisation s'accompagne généralement d'une ségrégation spatiale croissante (Bassand et al., op. cit.), elle reste peu marquée à Hanoï.

Pourtant, cette mixité sociale paraît paradoxale. Si les relations économiques entre les deux groupes sont fortes, les autres aspects de la vie quotidienne sont marqués par une séparation entre migrants et résidents. Dans les associations para-étatiques, comme l'Union des femmes, qui constituent un espace important de sociabilité à l'échelle locale, les migrants n’ont générale- 
ment pas leur place. Il n'y a pas d'équivalent pour les migrants : ces derniers ne semblent pas s'organiser en association formelle, il n'y a pas de constitution de groupe formalisé qui défendrait leurs intérêts ou favoriserait l'entraide. Les réseaux d'aide et de sociabilité sont souvent ségrégés : les migrants fréquentent d'autres migrants venus pour l'essentiel de leur région, voire de leur village d'origine (à l'exception, parfois, de relations d'amitié et d'entraide avec leur logeur), les résidents fréquentent des résidents. Ce clivage des réseaux de sociabilité et d'entraide est fondamental dans la construction de l'opposition entre « nous » et « eux », qui fonde l'identification des « migrants » comme minorité urbaine. La mixité spatiale atténue peu la ségrégation sociale.

\section{LES MIGRANTS COMME MINORITÉ DANS LA VILLE}

Par un processus d'étiquetage en tant que " migrants », la catégorisation administrative en vigueur construit un groupe social urbain dominé. En créant deux niveaux de citadinité et une citoyenneté différenciée, l'enregistrement résidentiel entérine, justifie et renforce les inégalités sociales et les rapports de domination entre les résidents et les migrants. Ces derniers se trouvent assignés à un statut social inférieur et l'enregistrement résidentiel institutionnalise une légitimité différenciée à être en ville.

Ce groupe se présente comme une minorité urbaine : un groupe défini par certaines caractéristiques (ici, l'origine rurale et l'impossibilité d'accéder au statut de résidents citadins), dont les membres sont moins nombreux que ceux qui ne partagent pas ces caractéristiques (ici, les « résidents » actuels et en devenir). Le statut administratif est le point de départ qui génère une typification des emplois, des conditions de vie, des réseaux sociaux participant à la définition du groupe. Cette identité est à la fois assignée par les « résidents » et acceptée, intériorisée par les « migrants » (qui utilisent eux-mêmes ce terme pour se nommer). Pourtant, elle recouvre des situations variées, elle regroupe des personnes sans affinité particulière (des migrants venant de provinces différentes ne se fréquentent pas nécessairement plus que des migrants et des résidents), et l'absence de relais institutionnel, de prise de position politique, et même de définition formalisée de ce qu'est un "migrant» conduit à l'absence de mobilisation du groupe en tant que tel. C'est une minorité en termes quantitatifs et avec des caractères propres qui la distinguent de la « majorité » résidente, mais non mobilisée, non revendiquée, non relayée, sans institutionnalisation, sans redéfinition propre du vocable « migrant ».

Le choix politique du maintien de l'enregistrement résidentiel renforce les inégalités sociales en milieu urbain. Il prolonge la volonté de l'État de contrôler le peuplement du pays, avec une souplesse qui permet une adaptation de la main-d'œuvre aux nouvelles demandes d'une économie en transition et de métropoles en croissance. Le groupe des migrants constitue ainsi un réservoir 
de main-d'œuvre mobile, flexible et bon marché qui permet de répondre à la demande et qui, grâce à la vie multi-locale, s'adapte aux fluctuations d'une économie de plus en plus ouverte sur le monde : lors du ralentissement économique de 2008-2009, le chômage en ville a peu augmenté, mais on a pu observer un retour - momentané - vers la campagne de travailleurs migrants. Le groupe des migrants assure à la fois un ensemble de services urbains à faible coût (récupération des déchets recyclables, transports de personnes, par exemple), tout en constituant un groupe à la fois mobile et flexible, sans relais politique ou social, qui se présente comme une variable d'ajustement à la croissance économique.

Finalement, cette étude met au jour la façon dont un dispositif administratif renforce des inégalités entre groupes sociaux au sein d'un pays où cellesci étaient faibles et croissent désormais très rapidement (Gironde, $2009^{10}$ ). La stratification sociale complexe, qui accompagne partout le processus de métropolisation, se traduit comme ailleurs par des rapports de domination, mais ils sont ici entérinés et légitimés par l'existence de cette catégorie de « migrants » qui mue un groupe relativement différencié en minorité urbaine.

\section{RÉFÉRENCES BIBLIOGRAPHIQUES}

Agergaard, J. ; Vu, T.T. 2010. « Mobile, Flexible, and Adaptable: Female Migrants in Hanoi's Informal Sector », Population, Space and Place, Wiley Online Library.

Bassand, M. ; Thai, T.N. Du ; Tarradellas, J. ; Cunha, A. ; Bolay, J.-Y. 2000. Métropolisation, crise écologique et développement durable. L'eau et l'habitat précaire à Ho Chi Minh-Ville, Vietnam, Lausanne, Presses polytechniques et universitaires romandes.

BRUNET, R. (sous la dir. de) 1992. Les mots de la géographie, dictionnaire critique, Paris, Reclus-La Documentation française.

CAstiglioni, F. ; Cusset, J.-M. ; Gubry, P. ; NGuYên, T.T. ; Pham, T.H. (sous la dir. de) 2006. La ville vietnamienne en transition, Paris, Karthala-IMV-PADDI.

CoRTĖs, G. 2000. Partir pour rester. Survie et mutation de sociétés paysannes andines, Paris, éd. ORSTOM-IRD

GIRONDE, C. 2004. «Les transformations de l'économie familiale dans le delta du Fleuve Rouge », dans C. Gironde et J.-L. Maurer (sous la dir. de) Le Vietnam à l'aube du XXI siècle. Bilan et perspective politiques, économiques et sociales,

10. L'Office général de la statistiques (GSO, www.gso.gov.vn) publie tous les deux ans une enquête sur les conditions de vie des ménages (Vietnam Households' Living Standards Survey) qui rend compte des revenus des ménages et de leur évolution. L'enquête de 2010 montre un accroissement des revenus du quintile le plus riche plus rapide que ceux du premier quintile ; le coefficient de Gini a passé le seuil de 0,4 au début des années 2000 et atteint aujourd'hui 0,43 à l'échelle du pays. 
Paris, Karthala. p. 193-227.

Gironde, C. ; MAurer, J.-L. (sous la dir. de) 2004. Le Vietnam à l'aube du XXI siècle. Bilan et perspective politiques, économiques et sociales, Paris, Karthala.

Gironde, C. 2009. «Réformes, croissance et augmentation des inégalités dans le delta du Fleuve Rouge - Viêt Nam (1980-2000)», Moussons, n 13-14, p. 269308.

Gubry, P. ; LÊ, T.H. ; Nguyên, T.T. ; PhAm, T.H. ; TrÂn, T.T.T. ; Vu, H.N. (sous la dir. de) 2008. Bouger pour vivre mieux. Les mobilités intra-urbaines à Hô Chi Minh Ville et Hanoi (Viêt-nam), Hanoi, Université nationale d'économie.

Gubry, P. ; LÊ, T.H. ; NGuYEN, T.T. 2011. «L'urbanisation au Vietnam : que sait-on de la 'population flottante'? », Séminaire : Population, santé publique et développement au Viêt-nam après 25 ans de Renouveau, Hanoï, IPSS, ARCUS, octobre 2011.

LABBÉ, D. ; Musil, C. 2011. «L'extension des limites administratives de Hanoi : un exercice de recomposition territoriale en tension », Cybergeo: European Journal of Geography, http://cybergeo.revues.org/24179

Lacoste, Y. (sous la dir. de) 1993. Dictionnaire de géopolitique, Paris, Flammarion.

LI, T. 1996. Peasants on the Move: Rural-Urban Migration in the Hanoi Region, Singapore, Institute of South East Asian Studies (ISEAS).

LI, Z. 2001. Strangers in the City. Reconfigurations of Space, Power, and Social Networks Within China's Floating Population, Stanford, Stanford University Press.

Luong, T.T.H. ; Nguyen, T.A. ; Rigg, J. ; Dinh, T.D. 2011. Becoming and Being Urban in Hanoi, The Discourse of the Rural-Urban Migrant, Rapport de recherche pour le projet « The Challenges of the Agrarian Transition in Southeast Asia », Social Sciences and Humanities Research Council of Canada.

OCDE. 2011. Examens de l'OCDE des politiques de l'investissement : Viet Nam 2009, OECD Publishing.

PAPIN, P. 2001. Histoire de Hanoi, Paris, Fayard.

PNud, 2010. Urban Poverty Assessment in Hanoi and Ho Chi Minh City, Hanoi.

Quertamp, F. 2010. «La périurbanisation de Hanoi. Dynamiques de la transition urbaine vietnamienne et métropolisation », Les annales de géographie, n 671 672, p. 93-119.

Racine, J.-L. (sous la dir. de) 1994. Les attaches de l'homme. Enracinement paysan et logiques migratoires en Inde du Sud, Paris, Maison des Sciences de l'Homme.

Thireau, I. ; Linshan, H. 2002. "À l'ombre des commerces en bordure de route », Études rurales $\mathrm{n}^{\circ} 161-162$, p. 109-127.

UAL www.cairn.info/revue-etudes-rurales-2002-1-page-109.htm

Vet -VIlles en tRAnsition, 2005. Impact of Existing Residence Registration Policy on Urban Poverty Alleviation, Hanoi, Institute of Sociology. 